\title{
Polímeros com Condutividade lônica: Desafios Fundamentais e Potencial Tecnológico
}

\author{
Virgínia P. R. Silva, Vinicius Caliman, Glaura G. Silva \\ Departamento de Química, UFMG
}

\begin{abstract}
Resumo: Polímeros condutores iônicos ou eletrólitos poliméricos constituídos por um sistema de sal dissolvido em uma matriz polimérica sólida são materiais que apresentam interesse científico e potencial tecnológico. A dissolução de sais em uma matriz polimérica amorfa ou semicristalina sólida leva a estudos sobre intrigantes aspectos estruturais, que podem ser abordados por técnicas físico-químicas diversas tais como RMN, Raman e Espectroscopia de Vida Média de Pósitrons. Os estudos estruturais são correlacionados com propriedades eletroquímicas visando à utilização desses materiais em dispositivos tais como baterias, supercapacitores e células solares. Grupos brasileiros têm gradativamente ampliado os estudos e aplicações de eletrólitos poliméricos sólidos.
\end{abstract}

Palavras-chave: Polímeros condutores iônicos, eletrólitos poliméricos, supercapacitores, baterias.

\section{Polymers with Ionic Conductivity: Fundamental Challenges and Technological Potential}

Abstract: Ionic conducting polymers or polymer electrolytes prepared with the addition of a soluble salt in a solid polymeric matrix are very important materials, associated with an intense research activity and technological efforts. Structural studies in a system of salt dissolved in an amorphous or semicrystalline solid polymeric matrix can be done with various techniques, such as NMR, Raman and Positron Annihilation Spectroscopy. The structural studies are correlated with electrochemical properties in order to evaluate these materials for applications in batteries, supercapacitors and solar cells. Brazilian researchers are contributing to the fundamental research and development of new applications of polymeric electrolytes.

Keywords: Ionic conductor polymers, polymeric electrolytes, batteries, supercapacitors.

\section{Introdução}

A produção, estocagem e distribuição de energia são algumas das maiores preocupações da sociedade contemporânea. O desenvolvimento de materiais para aplicações como eletrodo e eletrólito criou oportunidades para novos tipos de geração de energia e sistemas de estocagem, que podem vir a contribuir de forma decisiva para o desenvolvimento sustentável e harmônico da sociedade tecnológica ${ }^{[1]}$.

O uso de fontes inesgotáveis de energia alternativa como a energia solar e a eólica requerem sistemas de alto poder de estocagem para validar sua aplicação. Dentro dessa demanda, o estudo dos materiais condutores iônicos sólidos se revestiu de grande relevância. Pesquisas espaciais, criação de novos tipos de memória e arquitetura computacional, aparatos biomédicos e microsensores para controle de poluição atmosférica são algumas das áreas que se beneficiaram com o desenvolvimento destes materiais.

Os eletrólitos poliméricos contrastam agudamente com os condutores iônicos sólidos usuais baseados em cerâmicas como $\beta$-alumina sódica, vidros e cristais inorgânicos, no que diz respeito ao modo de transporte de carga e ao valor da condutividade iônica que é de 100 a 1.000 vezes menor, no caso dos polímeros ${ }^{[1]}$.

Este obstáculo é compensado por alguns fatores, por exemplo, os eletrólitos poliméricos podem formar filmes muito finos de alta área superficial conferindo altos valores de densidade de potência $\left(>100 \mathrm{~W} \mathrm{dm}^{-3}\right)$ aos dispositivos. Do ponto de vista eletroquímico, quando há uma mudança de volume durante os ciclos de carga e descarga, a célula eletroquímica baseada em eletrólitos poliméricos continua estável, sem a degradação observada nos materiais cristalinos ou vítreos, devido a tensões mecânicas. Outra vantagem seria que os eletrólitos poliméricos dispensam a necessidade de incorporar um separador inerte poroso na célula.

Já ao comparar os eletrólitos poliméricos sólidos com os sistemas eletrolíticos líquidos, como por exemplo, soluções de sais de lítio em solventes apróticos, pode-se afirmar que os líquidos apresentam um problema de instabilidade com relação à oxidação/redução dos solventes orgânicos em contato com o eletrodo, além de dificuldades com a volatilidade dos mesmos ${ }^{[2]}$.

A necessidade de um material de características plásticas como eletrólito levou, em 1978, Armand e col. ${ }^{[3,4]}$ a su-

Autor para correspondência: Glaura G. Silva, Departamento de Química , ICEx, UFMG, Caixa Postal 702, CEP: 31270-901, Belo Horizonte, MG. E-mail: glaura@zeus.qui.ufmg.br. 
gerir o uso de eletrólitos poliméricos como solventes sólidos para baterias recarregáveis de lítio, o polímero sugerido para esse fim foi o poli(óxido de etileno). Antes disso, a utilização de polímeros como matrizes para eletrólitos já havia sido sugerida no início dos anos 70 , quando Wright e col. ${ }^{[5-7]}$ relataram a formação de complexos entre poli(óxido de etileno) e tiocianato de potássio, nessa época a importância tecnológica da descoberta não foi completamente compreendida.

O polímero conhecido como POE - poli(óxido de etileno) foi estudado durante 20 anos como uma matriz de eletrólito polimérico. Entretanto, à temperatura ambiente, a utilização do POE como eletrólito polimérico se torna limitada por sua cristalinidade $^{[8]}$, o que impede a mobilidade iônica pelo enrijecimento das cadeias poliméricas. Muitas pesquisas têm buscado modificações na estrutura do POE com a intenção de alterar seu grau de cristalinidade à temperatura ambiente, estas envolvem, por exemplo, co-polimerizações, formação de blendas poliméricas ${ }^{[9]}$, introdução de plastificantes, etc.

Dessa forma o conceito de eletrólito polimérico foi ampliando-se e este termo, segundo F. Gray ${ }^{[10]}$, se refere na atualidade a materiais que se encaixem em qualquer umas das quatro categorias seguintes:

1) Um sistema solvente sólido, onde a fase condutora iônica é formada por um sal dissolvido em uma matriz formada por um polímero polar. Este é o eletrólito polimérico em sua descrição original.

2) Um eletrólito gel que é formado por um sal dissolvido em um líquido polar e tem a ele adicionado um polímero para promover estabilidade mecânica. O polímero seria uma espécie de matriz para a acomodação da solução. A necessidade de se obter eletrólitos poliméricos com boa condutividade à temperatura ambiente e abaixo desta levou à construção de eletrólitos géis ${ }^{[11]}$. Um exemplo pode ser dado por um sistema onde há a acomodação de uma solução de EC - PC (etileno carbonato - propileno carbonato) / $\mathrm{LiClO}_{4}$ em PAN - poli(acrilonitrila) ou PMMA - poli(metil metacrilato $)^{[12,13]}$. Além das matrizes PAN e PMMA, pode-se citar o PVC como matriz ${ }^{[12]}$.

3) Uma borracha iônica é formada por uma mistura de sal fundido a baixas temperaturas e uma pequena quantidade de polímero com alta massa molecular. Esta classe é muito parecida com os eletrólitos géis, do ponto de vista molecular. Um sistema de sais ternário formado por $\mathrm{LiClO}_{4}-\mathrm{LiNO}_{3}$ LiOAc, associado à pequenas quantidades de $\mathrm{POE}$ de alta massa molar, consiste em um exemplo desse tipo de eletrólito ${ }^{[14]}$.

4) Uma membrana ionômera, em particular, um condutor de prótons polieletrólito usado como eletrólito em membranas de troca protoiônicas (PEM) em células a combustível. Este material é também conhecido como eletrólito polimérico sólido para células combustíveis (SPEFC) e, normalmente, consiste em uma matriz que contém uma cadeia de flúorcarbono a qual grupos sulfônicos são acrescentados. Este eletrólito requer a presença de plastificantes, geralmente água, para ativar seus níveis de condutividade. Os materiais mais populares utilizados como membranas protoiônicas são ba- seados no ácido perfluorosulfônico, dentre eles o Nafion ${ }^{\mathrm{TM}}{ }^{[15]}$. A utilização dessas membranas vem enfrentando problemas com sua estabilidade a altas temperaturas, sugerindo a necessidade da busca por uma nova geração de materiais para utilização em células combustíveis ${ }^{[16]}$.

\section{Estrutura e Propriedades de Eletrólitos Poliméricos}

Os eletrólitos poliméricos referidos na categoria 1 são até hoje muito estudados e, por não possuírem condutividades muito altas, várias abordagens têm sido desenvolvidas para otimizá-los.

Um eletrólito polimérico da categoria 1 consiste em uma matriz polimérica contendo heteroátomos bons doadores de elétrons e um sal que seja solúvel na matriz sólida e apresente condutividade iônica pelo menos superior a $10^{-6}{\mathrm{~S} . \mathrm{cm}^{-1}}^{-}$. Para a obtenção de boas propriedades com um eletrólito polimérico desse tipo são necessárias no mínimo duas características ${ }^{[10]}$ :

- Baixas barreiras de rotação das ligações no segmento da cadeia polimérica;

- Uma distância adequada entre os centros de coordenação, promovendo múltiplas ligações intrapolímero;

Os sais preferenciais são sais de lítio com ânions do tipo $\mathrm{ClO}_{4}^{-}, \mathrm{CF}_{3} \mathrm{SO}_{3}{ }^{-}$entre outros, em virtude de maior solubilidade e condutividade iônica. Quanto às matrizes poliméricas, os poliéteres que contém o encadeamento -C-C-O-, desde que possuam uma estrutura predominantemente amorfa, preenchem perfeitamente os pré-requisitos acima.

De maneira geral o valor da condutividade iônica pode ser expresso de acordo com a equação $1^{[17,18]}$

$$
\sigma=\mathrm{nq} \mu
$$

onde $\mu$ é a mobilidade, $n$ é a concentração de portadores iônicos de carga e q é a carga dos íons. Portanto, o comportamento da condutividade iônica com o aumento da quantidade de sal dissolvido é influenciado por, pelo menos, três fatores:

I - O aumento da concentração de sal na amostra que, em geral, contribui para um aumento da condutividade, devido ao aumento do número de portadores de cargas;

II - A formação de interações do tipo íon-dipolo permanente entre o íon metálico do sal e os heteroátomos da cadeia polimérica (Figura 1-a), provoca um enrijecimento da cadeia e conseqüente aumento do valor da transição vítrea (Tg) do sistema. Isto leva a uma diminuição da condutividade pela perda da mobilidade. Neste contexto considera-se que a mobilidade iônica é dependente da mobilidade da cadeia polimérica, ou seja, o deslocamento do íon é assistido pelo polímero, como será discutido mais tarde neste texto.

III - Um aumento excessivo na concentração do sal provoca, um grande aumento na concentração de íons que podem interagir entre si formando pares iônicos ou agregados (Figura 1-b). Assim, uma menor quantidade de espécies efetivamente portadoras de carga ficaria disponível para promover a condução, diminuindo a condutividade.

As variações de entropia e entalpia devem ser considera- 


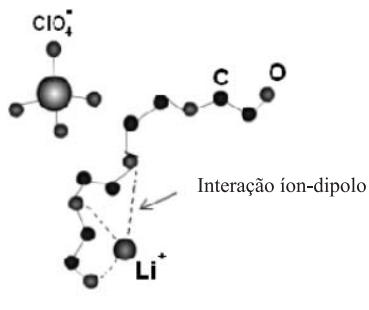

(a) (b)

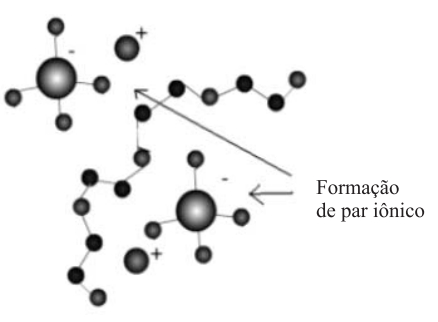

Figura 1 - Representação da interação entre uma cadeia polimérica tipo POE e o cátion (a) e formação de par iônico (b) em sistemas eletrólitos poliméricos.

das no processo de dissolução de um sal em um líquido ou sólido. A entalpia de solvatação de um sal em uma matriz polimérica depende grandemente da força de interação íondipolo, formada entre os cátions e os grupos constituintes da cadeia polimérica. A variação negativa na entalpia de solvatação, devido à formação dessas interações mais fortes é, de fato, o fator controlador do processo de dissolução do sal em um solvente polimérico ${ }^{[10]}$.

Todos os sistemas formados pela associação sal/polímero em um eletrólito polimérico sólido apresentam condutividade $^{[19]}$. À temperatura ambiente esse valor é cerca de duas ordens de grandeza menor do que nos líquidos orgânicos mas, entre $80^{\circ} \mathrm{C}$ e $120^{\circ} \mathrm{C}$ o valor da condutividade de um sistema eletrólito polimérico é cerca de $10^{-3} \Omega^{-1} \mathrm{~cm}^{-1}$, sem a contribuição de elétrons.

As medidas utilizando-se corrente alternada (a.c.) são as mais utilizadas para a determinação das propriedades elétricas dos eletrólitos poliméricos. Isto é devido, sem dúvida, à simplicidade das células com eletrodos bloqueadores que impedem a deposição do metal sobre o eletrodo. A técnica que utiliza essas medidas com corrente alternada é chamada Espectroscopia de Impedância e tem por mérito fornecer, de maneira sistemática, informações sobre a condutividade de um filme eletrólito sem a interferência de fenômenos de interface e sem alteração das propriedades do sistema pela $\operatorname{medida}^{[2,20]}$.

Em um experimento de impedância obtém-se diretamente a resistividade do eletrólito (R). Para o cálculo da condutividade usa-se a equação 2

$$
\sigma=(1 / A) \times(1 / R)
$$

onde lé a espessura da amostra, A é a área de contato amostra/eletrodo.

\section{Mecanismo de transporte iônico dentro de uma matriz polimérica}

Quando comparado a um eletrólito líquido, no eletrólito polimérico a matriz é o solvente e sua diferença em relação a um solvente líquido é a imobilidade, daí classificá-lo como condutor iônico sólido.

A Figura 2 mostra diferentes esquemas mecanísticos envolvendo o transporte de cargas iônicas. Em um solvente líquido, é necessário o deslocamento deste para que haja o processo de difusão dos íons solvatados. Qualquer tipo de transferência de carga através da interface de um eletrodo necessita de uma difusão também do solvente. Em um sólido cristalino, como, por exemplo, AgI, ou em estados vítreos há saltos entre posições na estrutura cristalina. Os íons permanecem em um poço potencial até receberem energia suficiente, através de ativação térmica, para romperem suas barreiras de energia e efetivamente se deslocarem. Assim, a condução nesses sólidos corresponde apenas ao movimento dos íons, uma vez que a matriz é estática ${ }^{[21]}$.

Os polímeros representam uma situação intermediária entre os sólidos cristalinos ou vítreos e os líquidos ${ }^{[21]}$. Neles, os sítios de solvatação estão covalentemente ligados na cadeia polimérica por ligações flexíveis. O deslocamento de cadeias poliméricas juntamente com os íons, situação encontrada nos líquidos, é praticamente impossível, especialmente quando tratamos de polímeros onde há reticulação entre as cadeias. Acima da Tg, os movimentos dos segmentos de cadeias poliméricas são comparáveis aos de um líquido ${ }^{[21]}$. Dessa forma, o mecanismo de condutividade tem origem em um processo de solvatação-dessolvatação ao longo das cadeias poliméricas. Quando o íon efetivamente se complexa ao sítio de solvatação, há uma separação entre as cargas iônicas onde cátion e ânion se movem em direções opos$\operatorname{tas}^{[10,19]}$

O POE, como dito anteriormente, foi a primeira matriz para eletrólito estudada, sua estrutura é de um polímero linear com unidades $\left(-\mathrm{CH}_{2} \mathrm{CH}_{2} \mathrm{O}-\right)$ e possui uma conformação em forma de hélice ${ }^{[2,10]}$. No final dos anos 70 , a condutividade

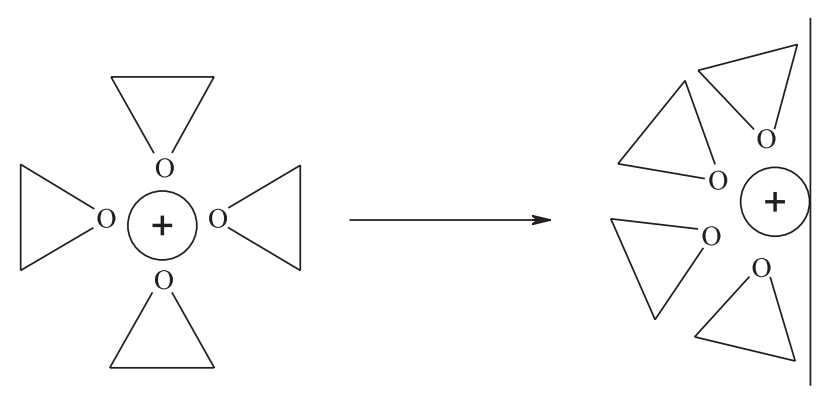

(a)

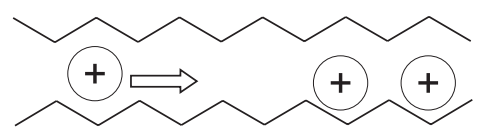

(b)

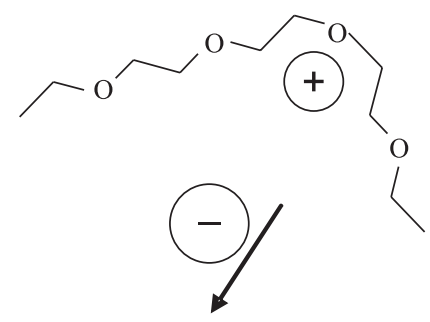

(c)

Figura 2. Modelos esquemáticos para mecanismos de condução iônica em líquidos (a), sólidos cristalinos ou vítreos (b) e polímeros (c) 
iônica de POE foi atribuída à passagem de cátions por uma espécie de túnel formado pela estrutura helicoidal na fase cristalina. Este mecanismo é similar ao adotado para condutores iônicos sólidos convencionais (Figura 2-b).

Estudos de Ressonância Magnética Nuclear foram, nesse ponto, de importante ajuda na elucidação do que realmente ocorria na mobilidade e conseqüente transporte iônico em uma matriz. Em $1983^{[22]}$, o uso do tempo de decaimento da função de correlação de magnetização nuclear transversa $\left(\mathrm{T}_{2}\right)$, analisando os núcleos na fase cristalina e amorfa, mostrou que em ambos os ambientes havia condutividade iônica. Com a verificação do aumento da condutividade com a temperatura em polímeros predominantemente amorfos, a indicação de que a fase amorfa era primordial para a mobilidade e transporte foi se confirmando ${ }^{[1,10]}$. Apesar dessa confirmação, não se pode afirmar que o mecanismo de difusão dos cátions por dentro das vacâncias da hélice do POE possa ser descartado, ainda hoje interessantes estudos debatem este tema ${ }^{[23]}$.

\section{Estudos físico-químicos de eletrólitos poliméricos}

É interessante ressaltar que além do potencial tecnológico desses materiais o estudo fundamental de estrutura e propriedades na área é muito intenso. O charme acadêmico desse sistema está associado ao fato de ser um sistema no estado sólido semicristalino ou amorfo, com arranjo complexo entre íons e matriz polimérica, influenciando diretamente as propriedades finais. Técnicas como RMN de sólidos e difração de raios X são de grande importância para a determinação da estrutura de um eletrólito polimérico e através dessas técnicas, os mecanismos de transporte puderam ser mais bem estudados ${ }^{[1,10]}$. Outras técnicas, além das anteriores, têm sido empregadas na elucidação de aspectos científicos intrigantes dos materiais eletrólitos poliméricos. Por exemplo, a Espectroscopia Raman e a EVMP - Espectroscopia de Vida Média do Pósitron são duas importantes ferramentas neste contexto.

\section{Espectroscopia Raman}

Ao se adicionar um sal à matriz polimérica observa-se que seus ânions, como por exemplo, $\mathrm{ClO}_{4}{ }^{-}$e $\mathrm{CF}_{3} \mathrm{SO}_{3}^{-}$, apresentarão vibrações moleculares com freqüências específicas de cada grupo ${ }^{[24-26]}$. Além disso, essas moléculas terão modos de vibração distintos se estiverem na forma de íons livres, pares iônicos ou agregados iônicos. Uma importante informação no estudo dos eletrólitos poliméricos seria determinar a forma de agregação na qual os ânions se encontram dentro da matriz após a dissolução.

Além dos modos de vibração associados aos ânions, podese também explorar modos da matriz polimérica que são modificados pela presença da estrutura de solvatação do cátion ${ }^{[24]}$. A espectroscopia Raman é uma poderosa ferramenta que tem sido usada com freqüência na determinação das características de vibrações associadas a cada tipo de interação iônica: íon-íon e íon-polímero ${ }^{[25,26]}$.

Podemos exemplificar esse tipo de estudo com as interações do ânion $\mathrm{ClO}_{4}^{-}$, onde um desdobramento da ban- da de freqüência $v_{1}$, referente ao estiramento simétrico de $\mathrm{ClO}_{4}^{-}$, no espectro Raman ocorre com o aumento da concentração de sal. Este comportamento tem sido cuidadosamente estudado por diversos autores ${ }^{[27,28]}$. Uma primeira banda em torno de $931-934 \mathrm{~cm}^{-1}$, associado aos íons livres é característica dominante no espectro de soluções diluídas. O par iônico, $\mathrm{M}^{+} / \mathrm{ClO}_{4}^{-}$pode ser associado a uma segunda banda que aparece em torno de $938 \mathrm{~cm}^{-1[29]}$. Em soluções mais concentradas novas bandas aparecem em freqüências mais altas indicando a presença de agregados iônicos na amostra.

No trabalho de R. A. Silva e col. ${ }^{[29]}$, utilizando micro Raman, foram investigados os diferentes tipos de complexação dos íons em matrizes de poliéteres de diferentes massas molares. Em poli(etilenoglicol) temos que para as massas molares maiores $(\mathrm{Mw}=6000)$ utilizadas, a complexação se dá preferencialmente pelos oxigênios da cadeia éter. Já para os polímeros com cadeias menores $(\mathrm{Mw}=200)$ foi observado que a complexação do íon, se dá preferencialmente através do grupo -OH terminal.

\section{EVMP}

A espectroscopia de vida média do pósitron (anti-partícula do elétron) é uma técnica sofisticada no estudo de polímeros. O seu princípio de funcionamento baseia-se na medida do tempo de vida das espécies positrônicas, isto é, mede-se o tempo decorrido entre o "nascimento" do pósitron (e espécies positrônicas) e seu desaparecimento ${ }^{[30]}$.

O pósitron, $p s$, é emitido através de uma fonte e pode aniquilar-se como pósitron livre ou formar pares que são as chamadas espécies positrônicas: orto-positrônio (o-ps) ou para-positrônio $(p-p s)$. O orto-positrônio é formado por um pósitron e um elétron com spins paralelos e possui um tempo de vida no vácuo em torno de $142 \mathrm{~ns}$, um valor considerado alto. Na matéria condensada o orto-positrônio pode, alternativamente, aniquilar-se com um elétron externo de spin oposto ao do pósitron. Esta aniquilação é chamada "pick-off", emite 2 fótons e possui um tempo de vida bem menor do que 142 ns, entre 1 e 3 ns. A medida de tempo de vida média útil em estudos estruturais de materiais é feita através do $o-p s$, uma vez que este valor para as demais espécies, $p$ - $p s$ e pósitron livre, é muito pequena em relação ao tempo de vida daquele.

O parâmetro de tempo de vida médio do o-ps é conhecido como $\tau_{3}$. A probabilidade de formação do $o-p s$, ou intensidade, seria um parâmetro associado à quantidade de espécies formadas no meio, e é conhecida como $\mathrm{I}_{3}$.

Como o processo de aniquilação depende fortemente das propriedades do meio em que ela se processa, a EVMP apresenta-se como uma técnica analítica extremamente poderosa, particularmente no que se refere a informações estruturais sub-nanométricas. O processo de aniquilação é sensível ao estado físico, mudanças de fase, teor de cristalinidade, propriedades físico-químicas tais como: tensão superficial, viscosidade, densidade, presença de vacâncias ou defeitos, volume livre, buracos, poros, e fenômenos de superfície ${ }^{[31]}$.

Em amostras poliméricas há uma quantidade grande de aniquilação "pick-off" e seus tempos de vida média variam 
entre 1-2 ns. Esses valores sugerem a existência de grandes cavidades na estrutura polimérica que são chamados volumes livres. De acordo com vários autores ${ }^{[17,31,32]}$, a espécie positrônica pode ficar aprisionada nesses volumes livres fazendo com que $\tau_{3}$ aumente muito.

Considerando os modelos de condutividade iônica em eletrólitos poliméricos ${ }^{[1,2,19]}$, espera-se que altas condutividades se relacionem a altos valores de $\tau_{3}$, ou seja, grandes volumes livres. Estes volumes livres são reorganizados através do movimento molecular que faz parte do mecanismo de transporte dos íons dentro da matriz.

Estudos feitos com o eletrólito poliuretana/ $\mathrm{LiClO}_{4}$ mostraram a correlação entre os volumes livres e a concentração de portadores de carga com a condutividade iônica ${ }^{[17]}$. $\mathrm{Li}^{+} \mathrm{e}$ $\mathrm{ClO}_{4}{ }^{-}$são espécies que não reagem com as espécies positrônicas, assim as variações observadas em $\tau_{3}$ podem ser relacionadas apenas à concentração e tamanho dos volumes livres. As medidas feitas por EVMP mostraram que a fração de volume livre do sistema diminui cerca de $40 \%$ com o acréscimo da concentração de sal entre $5 \%$ e $35 \% \mathrm{~m} / \mathrm{m}$ no eletrólito. Esses dados são coerentes com a diminuição da condutividade do eletrólito em concentrações mais altas de sal.

\section{Dispositivos Baseados em Eletrólitos Poliméricos Sólidos}

\section{Baterias de lítio}

O sistema proposto por Armand ${ }^{[19]}$ envolve um gerador recarregável sólido composto por dois eletrodos de lítio reversíveis. Um agindo como uma fonte de íons lítio durante a descarga, o outro sendo um receptor. Estes dois eletrodos são separados por um filme de eletrólito polimérico sólido (SPE) que age como um transportador dos íons lítio. Este processo é reverso durante a carga.

A utilização de filmes finos, de fato vem compensar a baixa condutividade dos eletrólitos e oferece muitas outras vantagens, por exemplo, baixa densidade de corrente e altos valores de potência.

A Tabela 1 apresenta uma comparação entre as baterias de lítio utilizando eletrólitos poliméricos (SPE-Li) e os sistemas comerciais mais comuns. Pode-se perceber que a relação entre o valor teórico e o prático obtido para a densidade de energia da bateria feita com o eletrólito polimérico sólido (1/4) supera os valores obtidos para os demais sistemas, ou seja, Pb-ácido (1/8) e Ni-Cd (1/7). É também importante

Tabela 1. Características das baterias SPE-lítio frente às baterias comerciais mais utilizadas, $\mathrm{Pb}$-ácido e $\mathrm{Ni}-\mathrm{Cd}^{[33]}$

\begin{tabular}{|c|c|c|c|}
\hline \multirow{2}{*}{$\begin{array}{l}\text { Sistema/Tipo } \\
\text { de bateria }\end{array}$} & \multicolumn{2}{|c|}{$\begin{array}{c}\text { Densidade de energia } \\
\left.\text { (Wh. } \mathrm{Kg}^{-1}\right)\end{array}$} & \multirow{2}{*}{$\begin{array}{c}\text { Temperatura } \\
\text { de operação } \\
\left({ }^{\circ} \mathrm{C}\right)\end{array}$} \\
\hline & Teórica & Prática & \\
\hline Pb-ácido & 250 & $\sim 25-35$ & -20 a 40 \\
\hline Ni-Cd & 245 & $\sim 35$ & -40 a 50 \\
\hline SPE - lítio & 480 & 120 & -10 a 130 \\
\hline
\end{tabular}

salientar que não há reação secundária no processo eletroquímico envolvendo baterias SPE-lítio, o que não ocorre nos demais sistemas onde há uma reação secundária de formação de água. O tempo de vida útil de uma bateria SPElítio se iguala ao tempo de uma bateria $\mathrm{Ni}-\mathrm{Cd}$ e ambos são superiores ao tempo de vida útil de uma bateria $\mathrm{Pb}$-ácido ${ }^{[33]}$.

Apesar das vantagens apresentadas pela bateria SPE-lítio, em relação aos demais sistemas comumente usados, existem alguns obstáculos a serem superados para a comercialização dos dispositivos. O aumento da condutividade dos eletrólitos poliméricos sólidos, obtido através do esforço de duas décadas de trabalho, não têm sido suficiente para permitir seu uso à temperatura ambiente ${ }^{[34]}$.

\section{Supercapacitores ou capacitores de dupla camada elétrica}

Um capacitor de dupla camada ou supercapacitor é baseado na separação de carga interfacial através de uma dupla camada elétrica ${ }^{[35]}$, formada na interface eletrodo/eletrólito ou ao redor de partículas ou moléculas de carbono dispersas no eletrodo. Com eletrodos de alta área superficial a capacitância por unidade geométrica é amplificada pelo fator de rugosidade que pode atingir um valor de $10^{5}$. Supercapacitores baseados neste conceito de estocagem de energia tem sido propostos para diversos usos como, por exemplo, telefones celulares, computadores portáteis, terminais de dados "sem cabos", sistemas automotivos de partida e veículos híbridos e elétricos ${ }^{[36]}$.

As faixas de densidade de energia e potência para os supercapacitores, comparadas àquelas para as baterias chumbo-ácido, geralmente utilizadas nos automóveis, estão apresentadas na Tabela 2.

Portanto, supercapacitores são dispositivos de alto desempenho em relação à densidade de potência.

A evolução da sua tecnologia, entretanto, está associada à evolução dos materiais constituintes como, por exemplo, o uso de eletrólitos poliméricos na forma de filmes no lugar dos eletrólitos líquidos. Em relação ao eletrodo de carbono, podese dizer que os pós de carbono amorfo (negro de fumo) e as mantas ou tecidos porosos competem em descrições diferentes de produtos ${ }^{[38,39]}$. Mais recentemente os nanotubos de carbono têm sido alvo de interesse com patentes depositadas ${ }^{[40]}$. Dentre os grandes desafios, para se conseguir na prática o desempenho previsto teoricamente ( $300 \mathrm{~F} / \mathrm{g}$ carbono) para esses dispositivos, estão controlar a área superficial e a superfície condutora, por exemplo, das partículas nanométricas de

Tabela 2. Características elétricas de supercapacitores e baterias chumboácido ${ }^{[37]}$

\begin{tabular}{lcc}
\hline & $\begin{array}{c}\text { Densidade de energia } \\
\left(\mathbf{K J} . \mathbf{K g}^{-1}\right)\end{array}$ & $\begin{array}{c}\text { Densidade de } \\
\text { potência } \\
\left(\mathbf{K W} . \mathbf{K g}^{-1}\right)\end{array}$ \\
\hline Supercapacitores & $3,6-18$ & $0,3-1,0$ \\
$\begin{array}{l}\text { Baterias } \\
\text { chumbo-ácido }\end{array}$ & $54-108$ & $0,1-0,3$ \\
\hline
\end{tabular}


carbono amorfo ou nanotubos de carbono e garantir boa dispersão dessas entidades na matriz de eletrólito polimérico.

O grupo que trabalha com eletrólitos poliméricos na UFMG tem se dedicado ao estudo dos materiais constituintes e do supercapacitor. Alguns resultados sobre o dispositivo completo já foram publicados ${ }^{[41,42]}$

\section{Pesquisa em Eletrólitos Poliméricos no Brasil}

Resultados recentes de grupos brasileiros mostram que materiais eletrólitos poliméricos têm sido estudados de um ponto de vista fundamental ${ }^{[33-45]} \mathrm{e}$ aplicado ${ }^{[46-48]}$. Além dos supercapacitores ${ }^{[41,42]}$ já mencionados, há desenvolvimento de baterias ${ }^{[47]}$ e células solares ${ }^{[48,49]}$ com tecnologia nacional.

\section{Agradecimentos}

Os autores agradecem a agência de fomento CNPq. Um agradecimento especial é dedicado aos colaboradores do grupo de polímeros do Departamento de Química da UFMG que vem atuando no tema eletrólitos poliméricos há 12 anos: José Caetano Machado, Jean Michel Pernaut (in memoriam) e Clascídia Aparecida Furtado.

\section{Referências Bibliográficas}

1. Gray, F. M. - "Solid Polymer Electrolytes/Fundamentals and technological applications", VCH Publishers Inc., Cambridge (1991).

2. Armand, M. B. - "Current State of PEO-based electrolytes", in: Polymer Electrolyte Reviews - 1, cap.1, Eds. J. R. MacCallum \& C. Vincent, Elsevier: London (1987).

3. Armand, M. B.; Chabagno, J. M. \& Duclot, M. J., in: Second International Conference on Solid Electrolyte: St. Andrews, Scotland (1978).

4. Armand, M.; Chabagno, J. M. \& Duclot, M. J. - "Fast Ion Transport in Solids", Eds. Vashishta, P., Mundy, J. N. \& Shenoy, G. K, North Holland, Amsterdan, (1979).

5. Fenton, D. E.; Parker, J. M. \& Wright, P. V. - Polymer, 14, p.589 (1973).

6. Wright, P. V. - Br. Polym. J., 7, p.319 (1975).

7. Wright, P. V. - J. Polymer Sci., Polymer Phys. Ed., 14, p.955 (1976).

8. Allcock, H. R.; Olmeijer, D. L. \& O’Connor, S. J. M. Macromolecules, 31, p.753 (1998).

9. Nekoomanesh, M. H.; Wilson,D. J.; Booth, C. \& Owen, J. R. - J. Materials Chem., 4(12), p.1785 (1994).

10. Gray, F. M. - "Polymer Electrolytes", RSC Materials Monographs, Cambridge (1998).

11. Pistoia, G.; Antonini, A. \& Wang, G. - J. Power Sources, 58, p.139 (1996).
12. Ostrovskii, D.; Torell, L. M.; Appetecchi, G. B. \& Scrosati, B. - Solid State Ionics, 19, p.106 (1998).

13. Johansson, P.; Edvardsson, M.; Adebahr, J. \& Jacobsson, P. - J. Phys. Chem. B, 107, p.12622 (2003).

14. Fan, J. \& Angell, C. A. - Electrochim. Acta, 40, p.2397 (1995).

15. Panero, S.; Ciuffa, F.; D’Epifano, A. \& Scrosati, B. Electrochim. Acta, 48, p.2009 (2003).

16. Zhou, X.; Weston, J.; Chalkova, E.; Hofmann, M. A.; Ambler, C. M.; Allcock, H. R. \& Lvov, S. N. Electrochim. Acta, 48, p.2173 (2003).

17. Furtado, C. A. Silva, G. G.; Machado, J. C.; Pimenta, M. A. \& Silva, R. A. - J. Phys. Chem. B, 103, p.7102 (1999).

18. Ferry, A.; Oradd, G. \& Jacobsson, P. - J. Chem. Phys., 108(17), p.7426 (1998).

19. Armand, M. B. - Adv. Materials, 2, p.278 (1990).

20. Bruce, P. G. - "Electrical Measurements on Polymer Electrolytes" in: Polymer Electrolyte Reviews - 1, cap. 8, Eds. J. R. MacCallum, \& C. Vincent, Elsevier: London (1987).

21. Armand, M. B. - Solid State Ionics, 69, p.309 (1994).

22. Berthier, C.; Gorecki, W.; Minier, M.; Armand, M. B., Chabagno, J. M. \& Rigaud, P. - Solid State Ionics, 11, p.91 (1983).

23. Gadjourova, Z.; Andreev, Y. G.; Tunstall, D. P. \& Bruce, P. G. - Nature, 412, p.520 (2001).

24. Ferry, A.; Jacobsson, P. \& Torrel, L. M. - Electrochim. Acta, 40, p.2369 (1995).

25. Watanabe, M.; Ikeda, J. \& Shinohara I. - Polym. J., 15, p.65 (1983).

26. Torrel, L.M. \& Schantz, S.- "Light Scattering in Polymer Electrolytes", in: Polymer Electrolyte Reviews - 2, cap. 1, Eds. J. R. MacCallum, \& C. Vincent, Elsevier: London (1989).

27. Chabanel, M.; Legoff, D. \& Touaj, K. - J. Chem. Soc., Faraday Trans., 92, p.4199 (1996).

28. Silva, R. A.; Silva, G. G. \& Pimenta, M. A. - Appl. Phys. Lett, 67, p.3352 (1995).

29. Silva, R. A.; Silva, G. G. \& Pimenta, M. A. - Electrochim. Acta, 46, p.1687 (2001).

30. Mogensen, O. E. - "Positron Annihilation in Chemistry", Springer-Verlag, Berlim (1995)

31. Bamford, D.; Dublek, G.; Reiche, A.; Alam, M. A.; Meyer, W.; Galvosas, P. \& Rittig, F. - J. Chem. Phys., 115(15), p.7260 (2001). 
32. Forsyth, M.; Sun, J.; MacFarlane, D. R. \& Hill, A. J.; - J. Polym. Sci., Part B: Polym. Phys., 38, p.41 (2000).

33. Gauthier, M; Belanger, A; Kapfer, B; Vassort, G \& Armand, M. - "Solid Polymer Electrolyte Lithium Batteries", in: Polymer Electrolyte Reviews - 2, cap.9, Eds. J. R. MacCallum \& C. Vincent, Elsevier: London, (1989).

34. Tarascon, J. M. \& Armand, M. - Nature, 414, p.359 (2001).

35. Conway, B. E. - "Electrochemical Supercapacitors Scientific Fundamentals and Technological Application", Kluwer Academic/Plenum Publishers, Londres (2001).

36. http://www.cap-xx.com

37. Breakthrough in Supercapacitors - Skeleton Technologies Group: http://www.skeleton-technologies.com

38. Kawamoto, H. - "Carbon Black - Polymer Composites" in: E.K. Sichel (ed.), Marcel Dekker Inc., New York (1982).

39. Tanahashi, I.; Yoshida, A. \& Nishino, A. - J. Electrochem. Soc. 137, p.3052 (1990).

40. Lee, Y. et al - United States Patent, 6,454,816, Set. (2002).
41. Furtado, C. A.; Souza, P. P.; Silva, G. G.; Matencio, T. \& Pernaut, J. M. - Electrochim. Acta, 46, p.1629 (2001).

42. Pernaut, J. M. \& Goulart, G. - J. Power Sources, 55, p.93 (1994).

43. Rocco, A. M.; Fonseca, C. P.; Loureiro, F. A. M. \& Pereira, R. P. - Solid State Ionics, 166(1-2), p.115 (2004).

44. Bloise, A. C.; Donoso, J. P.; Magon, C. J.; Rosário, A. V. \& Pereira E. C. - Electrochim. Acta, 48, p.2239 (2003).

45. Cruz, A. T.; Silva, G. G.; Souza, P. P.; Matencio, T.; Pernaut, J. M. \& De Paoli, M. A. - Solid State Ionics, 159(3-4), p.301 2003.

46. Neves, S. \& Fonseca. C. P. - J. Braz. Chem. Soc., 15(3), p.395 (2004).

47. Montoro, L. A.; Rosolen, J. M.; Shin, J. H. \& Passerini, S. - Electrochim. Acta, 49, p.3419 (2004).

48. Longo, C. \& De Paoli, M. A. - J. Braz. Chem. Soc., 14(6), p.889 (2003).

49. Longo, C.; Nogueira, A. F.; De Paoli, M. A. \& Cachet, H. - J. Phys. Chem. B, 106(23), p.5925 (2002).

Enviado: 26/04/05

Aprovado:05/08/05 